\title{
Automotive Metal Material Database Development and Application in Vehicle Design
}

\author{
MENG Xianming ${ }^{1}$, ZHANG Sai ${ }^{1}$, FANG Rui ${ }^{1}$ and ZHU Yaohui ${ }^{2}$ \\ 1. China Automotive Technology \& Research Center, Tianjin 300300, China \\ 2. ARUP, Shanghai 200031, China
}

\begin{abstract}
This paper introduced the metal material database development and usage in car body design for automatic process. According to the common automotive steel product from the major steel plant in China and usage situation in local automotive OEMs (original equipment manufacturers), a standard LS-DYNA material database contains 129 kinds of commonly used metal material and database is developed by CATARC. Considering the frequent use of material data and applied to different parts in FE model, engineers can save time with this standard tool and meet the future request of the automation modeling process.
\end{abstract}

Key words: Metal material, CATARC database, structure design.

\section{Introduction}

The passive safety is an important performance of vehicle design, and it attracts more and more attention of the management institutes, OEM and consumer. Along with design capability improvement, various mandatory regulations and consumer evaluation systems keep on adding new test methods and harsher test evaluation, such as NCAP, RCAR, ADAC, IIHS. The more difficult test evaluation forces more difficult vehicle design requirements.

In the past 30 years, computer technology has taken a good progress. Accompanied by this, the CAE analysis technology received a high speed development, which results in CAE technology translating from the design assistant role to the design dominated role. However, the experiments have been faded to a verification means. The Euro-NCAP system has brought CAE analysis which results into the scoring system. So the CAE analysis accuracy in the design phase not only influences the design cycle, cost, performance, but also the NCAP scored directly.

At this stage, vehicle manufacturers also put forward

Corresponding author: MENG Xianming, Ph.D., senior engineer, research field: automotive material mechanical properties research. higher requirements of vehicle passive safety performance design which is driven by CAE. For instance, saving CAE model pre-processing time as much as possible, developing standard modeling methods to ensure that both new employees and experienced engineers can make the same results, generating standard reports, and so on. In this way, engineers do have more time to solve the real engineering problems. The trend of vehicle passive safety CAE analysis is a result of the modeling standardization, process automation and post-processing automation.

In the process of establishing the CAE model, the grid, connection, analysis and other conditions can be automated under certain standards, but the material parameters tend to be bottlenecks which affect the accuracy of the analysis results mostly. The same structure can use different material models to make a completely different result. And materials, especially metal materials, are quite different from others. In China, for example, hundreds of OEMs purchase metal raw materials mainly from Baosteel, Wuhan Iron and Steel and several other steel mills. If we can establish a standard database based on the commonly used automotive materials for passive safety CAE analysis, 
you can significantly improve the accuracy and consistency of CAE analysis results.

After two years of experiment, China automotive engineering technology and Research Center Automobile Engineering Research Institute established a material dynamic mechanical property database based on the passive safety CAE analysis software LS-DYNA

\section{MAT24_PIECEWISE_LINEAR_PLASTICITY}

constitutive model. In this database, 129 kinds of Hebei iron and steel, Baosteel, Wuhan Iron and Steel, Shougang, Anshan Iron and Steel and other commonly used automotive metal materials are included, and these databases have been integrated into Oasys/Primer, to meet the modeling process and automation requirements.

\section{General Treatment Method of Metal Material Model}

The passive safety crash test covers a range from low speed of $5 \mathrm{~km} / \mathrm{h}$ to high speed of $80 \mathrm{~km} / \mathrm{h}$, while metal materials generally exhibit strain rate effects, i.e., the stress-strain relationship of the material after yield is related to the rate of strain, as shown in Fig. 1.

Under normal circumstances, the material stress-strain relationship data mainly were obtained from the physical experiments, commonly used in uniaxial tensile experiments, Hopkins rod experiments and so on. For example, a uniaxial tensile, as shown in Fig. 2.

The strain is:

$$
\varepsilon=\frac{\Delta L}{L_{0}}
$$

The corresponding strain rate is:

$$
\dot{\varepsilon}=\frac{d \varepsilon}{d t}
$$

In LS-DYNA software, the material with the strain rate-dependent constitutive equation is MAT_24. This material model allows the user to input a set of curves of stress-strain relationship at different strain rates and is defined as * DEFINE_TABLE. It is important to note that curves in the TABLE must be the relationship between real stress and plastic strain, namely:

True stress $=$ engineering stress $\times$

$$
\text { ( } 1+\text { engineering strain) }
$$

Plastic strain $=\log (1+$ engineering strain $)$

At the same time, MAT_24 material requires the curves must be monotonically risen, it leads to the data which cannot be used after the material necking happened. So further processing of the experiment data needs to be done. Fig. 3 shows the curves treatment, respectively:

(1) Find the yield point of the material in the real stress and strain curve;

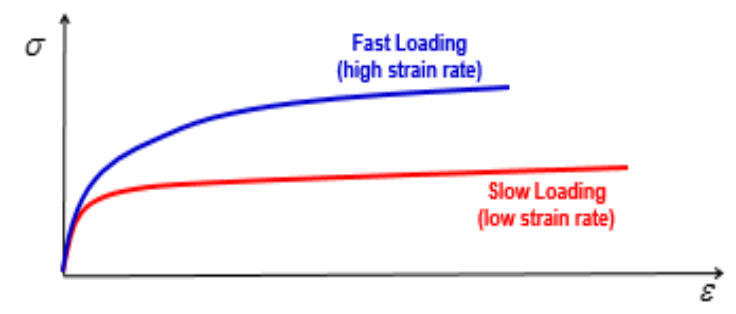

Fig. 1 Strain rate effect on metal material.

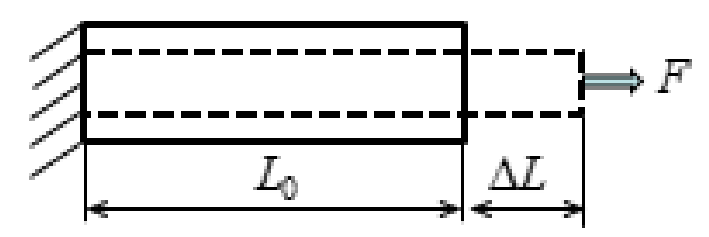

Fig. 2 Schematic diagram of uniaxial tension.

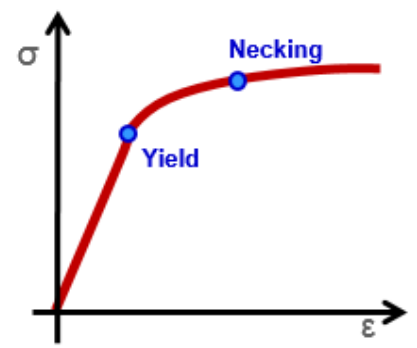

(a) True stress and strain

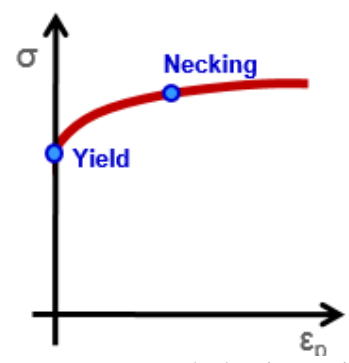

(b) True stress and plastic strain

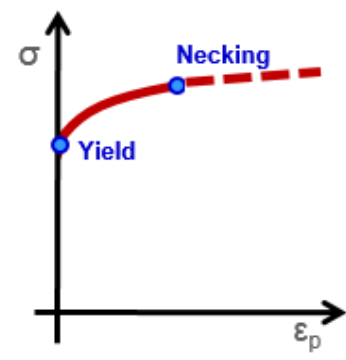

(c) True stress and plastic strain (after necking)

Fig. 3 General processing of metal materials stress-strain relationship. 
(2) Kick out the data before yield and make the $\mathrm{x}$-axis to zero, translate the curve into a real stress and plastic strain curve;

(3) Find the neck point and extend the curve after this point to a sufficient range, the general recommendation to extend to $200 \%$ of the position.

\section{CATARC Material Database}

The above general processing method [1] is an effective and basic processing rule of metal material model, but in practical applications, it will encounter various problems and have inaccurate factors, such as:

(1) Curves' consistency with the same strain rate is not good;

(2) The yield point is difficult to determine;

(3) Necks are difficult to determine how to extend;

(4) Under the action of external forces, different types of steels are corresponding to different deformation mechanisms and strain hardening mechanism, such as duplex steel, ferrite steel, TWIP steel, TRIP steel and so on. So for each type of steels, it has corresponding constitutive models;

(5) The strain rate and space, as well as strain rate and time, have asymmetry in dynamic formation process. So it needs to calibrate the true strain rate of the material and the true yield strength and rheological stress characteristics of the material in the parallel section of the specimen.

Another important problem which is always easily overlooked is that the above processings are based on the constant strain rate of the material in the same tensile test, but in fact this involves many factors, the state of the sample, the accuracy of the test equipment, and the measurement method.

CATARC developed a variety of metal materials covering more than 129 kinds of metal materials dynamic mechanical database, which is based on the domestic OEM widely used vehicle metal materials grades (HBIS, Baosteel, Shougang, Wuhan Iron and Steel, Anshan Iron and Steel and other steel companies). Data in this database are obtained by a series of data processing, simulation analysis, calibration and component calibration. It has been applied in the design of a variety of vehicles, and the late actual vehicle collision results and the previous CAE analysis results are basically the same. The material database not only achieved high CAE analysis precision requirements, but also saved a lot of time and cost for the vehicle CAE process.

In the next, the paper will do a brief description of the calibration processing methods of material dynamic mechanical property curves in the material database. There are many methods to smooth and extend the material dynamic mechanical properties data, but the most commonly used methods are JC equation, CS equation, ZA equation, PA equation etc. These conventional equations have relevant mechanical constitutive parameters and modified parameters, but the application of these equations has same limitations. Because of the material organization characteristics variety, different organizational characteristics correspond to different deformation mechanisms [2], and different reinforcement mechanisms. In the high-speed deformation process, different organizational properties correspond to the different flow stress state and strain hardening parameters, it cannot cover the mechanical constitutive of all materials just using a same constitutive. This database avoids the simplicity of the above methods perfectly, it classifies the vehicle metal materials into different type according to organization style and reinforcement mechanisms: single-phase steel, alloy steel, TWIP steel, TRIP steel, QP steel, manganese boron hot-formed steel, etc. Based on these major categories, the database corresponds to different materials and different constitutive models, and the relevant parameters of the constitutive model are based on the stress three-axis and micro-organizational properties of the material, which can better react the mechanical state of the material and its own mechanical properties.

After the initial data processing, the CAE data 
re-calibration process is performed. In Fig. 4, the left figure shows the calibration model of the material unidirectional tensile. Figure on the right shows the material dynamic curves after initial processing which will be input into the model. Because at a tensile rate, the various parts of the parallel section have different tensile speed, we do have to enter the whole strain rates range curve group into the calibration model.

After the basic model is constructed, the initial conditions and the relevant boundary conditions of the model are established. The lower ended contact of the sample is used as the power point of the dynamic tensile test. The sample is subjected to high-speed tensile to make a deformation. The displacement boundary conditions are also loaded in the lower end of the contact, ordering to maintain consistent with the test state. The top of the specimen is the fixed end and also keeps in the same state with the test. The input data of the lower contact in the model are shown in the figure on the right of Fig. 5. The input curve is the time and displacement curve recorded by the high speed test machine.

Fig. 6 shows the calculated strain rate-time curves (shown by the red curve) at different positions of the corresponding dynamic sample. The four graphs on the right of Fig. 6 are corresponded to point 1, point 2, point 3 and point 4 , successively. From the four graphs on the right, it can be seen that the strain rates at the four point positions vary with time and are not a constant. Therefore, for the strain and stress curve tested by the experiment, its strain rate is not accurate and needs to be calibrated again. In addition, it can be seen further from the four graphs, the trend of the relationship between the strain rate and the time curve of each measurement point is completely different. So it can be obtained that the strain rates are spatial heterogeneity in the parallel section of the tensile specimen. But in the uniaxial tensile test, the test is carried to obtain the mechanical properties of the parallel section, therefore, the relationship between strain rate and space needs to be analyzed. For the important mechanical parameter values in the simulation analysis - the yield point of the material, seen from the four graphs, the experiment strain rate is not the same with the strain rate corresponded to the yield point, so it needs to be re-calibrated and reanalyzed.

After the above test sample calibration process, the material model is built. Here is a list of some kinds of material curve models contained in the material database.

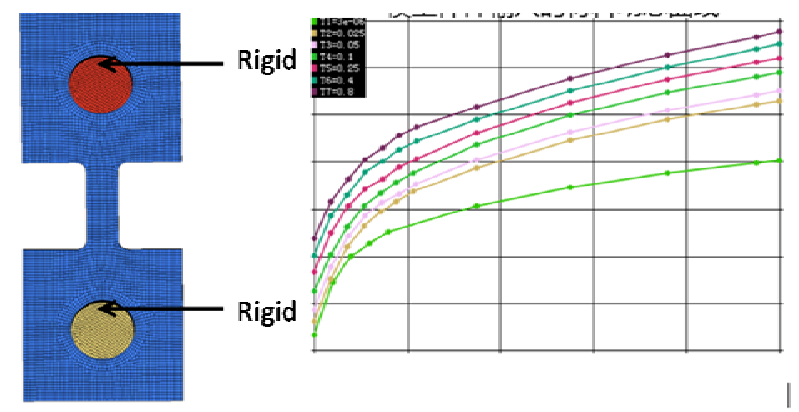

Fig. 4 Material calibration model and input dynamic mechanical curve group.

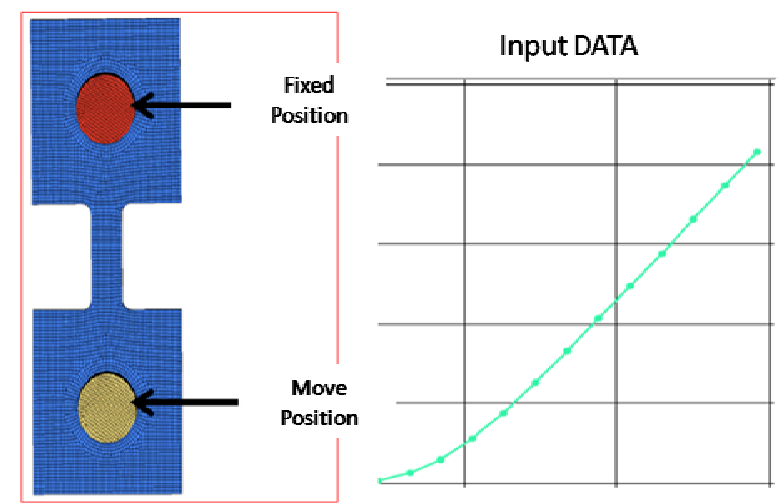

Fig. 5 Material calibration model and its boundary input conditions.

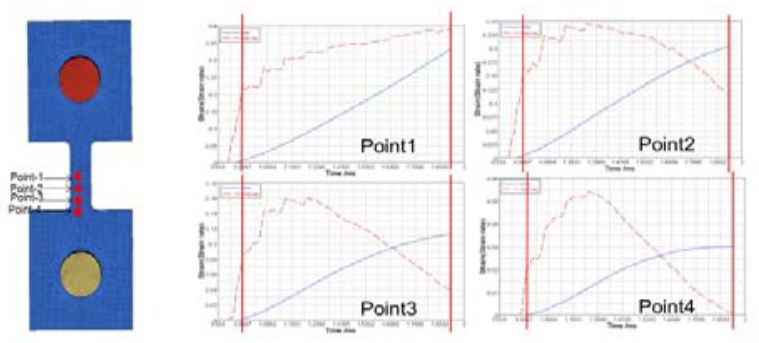

Fig. 6 Model strain rate analysis results. 
Table 1 CATARC material database.

\begin{tabular}{llll}
\hline Hebei Iron and Steel & & \multicolumn{2}{c}{ Baosteel } \\
\hline B170-1.6 mm & 22MnB5-1.0 mm & \multicolumn{1}{c}{ B340LA-1.5 mm } & DC06-1.0 mm \\
DC01-0.8 mm & ASTM1011A-1.8 mm & HC300LAD + Z100MB-1.2 mm & \\
DC01-0.8 mm & B170P1-0.7 mm & B410LA-1.6 mm & DC54D + Z-1.0 mm \\
DC01-2.0 mm & B210-1.6 mm & DC01-1.2 mm & HC-220BD + Z-0.9 mm \\
DC03-1.6 mm & B250LA-1.5 mm & DC01-1.6 mm & DC52D + Z-0.7 mm \\
DC04-1.0 mm & B250P1-0.8 mm & DC03-1.2 mm & HC340 - 590DP-1.2 mm \\
DC04-1.5 mm & B250P1-1.0 mm & DC04-0.6 mm & Q195-1.5 mm \\
DC04-2.0 mm & B250P1-1.2 mm & DC04-0.7 mm & Q195-2.0 mm \\
DC05-1.2 mm & B250P1-2.0 mm & DC04-1.6 mm & SAPH440-1.6 mm \\
DC06-1.2 mm & B280VK-1.9 mm & DC05-0.9 mm & SPCC-1.0 mm \\
HC220Y-1.0 mm & B340-590DP-1.0 mm & DC05-1.0 mm & SPFH590-1.6 mm \\
HC260LA-1.2 mm & B340-590DP-1.5 mm & DC06-0.7 mm & STKM11A-1.5 mm \\
SAPH440-P-1.6 mm & B340LA-1.2 mm & DC06-0.8 mm & B340LA-1.6 mm \\
\hline
\end{tabular}

4. Material Database Management and Application

As a standard database, shown in Table 1, the consistency of the model and the convenience of using are critical, at the same time, considering the automation requirements of the vehicle crash modeling process, here only use material database in the Oasys/Primer to manage.

\subsection{Material Database Establishment Method}

Oasys/Primer can establish a standard material database through the database file and index file to manage the material data and curve data. Fig. 7 is Primer database file structure.

The database file [4] is a standard file named oa-database and is stored in the OASYS software installation directory \$ OASYS or the user directory $\$$ HOME. It contains the database type, database storage location, and database name. And we can build a variety of different databases in the database file.

The database file indicates the Primer software detailed data file storage, location and name information. For examples, establish three databases:

\$ Database file for Primer in \$OASYS

\$ Any databases which are defined in here will be available to all users

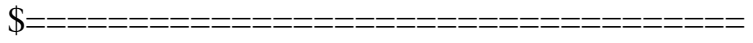

MATL* D:ICATARC_Material_Database\Ansteel CATARC Ansteel

MATL* D:ICATARC_Material_DatabaselBaosteel CATARC Baosteel

MATL* D:IMAT_DatabaselMAT_Steel ARUP

The index file named in the standard format as oa_index, contains the specific material data structure information, and is stored in the directory of each material database, such as D: ICATARC_Material_Database\Ansteel. The index file contains three types of information, including file name, material name, and material type, as follows:

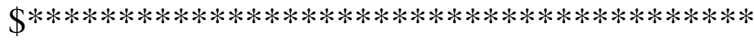

3

$\$ * * * * * * * * * * * * * * * * * * * * * * * * * * * * * * * * * * * * * * * * *$

Filename

Material Name

LS_DYNA Material Type

$\$ * * * * * * * * * * * * * * * * * * * * * * * * * * * * * * * * * * * * * * * *$

B170-1.6 mm.k

B170-1.6 mm

PIECEWISE_LINEAR_PLASTICITY

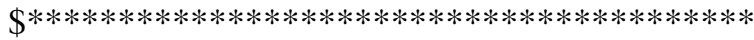

B210-1.6 mm.k

B210-1.6 mm

PIECEWISE_LINEAR_PLASTICITY

$\$ * * * * * * * * * * * * * * * * * * * * * * * * * * * * * * * * * * * * * * * *$

DC01-1.2 mm.k 
DC01-1.2 mm

\section{PIECEWISE_LINEAR_PLASTICITY}

$\$ * * * * * * * * * * * * * * * * * * * * * * * * * * * * * * * * * * * * * * * *$

At the end, save each material as a separate file in the corresponding database files, and it's available for application.

\subsection{Material Database Use}

There are two ways to use material models. The one is creating a new component and selecting a corresponding material model. Another way is giving all of the material models to their corresponding components at once while the vehicle crash model is first established. Here are the two methods.

The first way creates a new material [6] in Primer and then selects import from database. Primer will display the default database, engineers can choose the corresponding material by name, as shown in Fig. 8, using CATARC Angang B170-1.6 mm material. Primer will input all the information into the primer model based on the index file.

If you need other database information, just select another database directly, as in Fig. 9.

The second way is in order to meet the needs of automation, primer has developed a function which can match and give corresponding components material properties according to their material name automatically. First of all, inputting the established model's corresponding BOM table into model through the BOM function, including part name, material name and thickness and other basic parts information, as shown in Fig. 10.

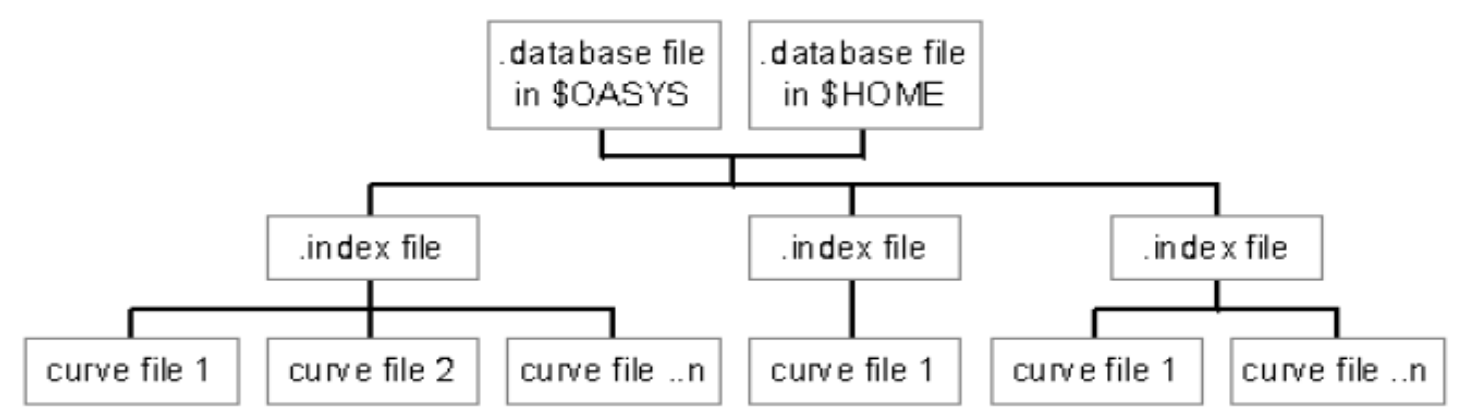

Fig. 7 Primer database file structure.

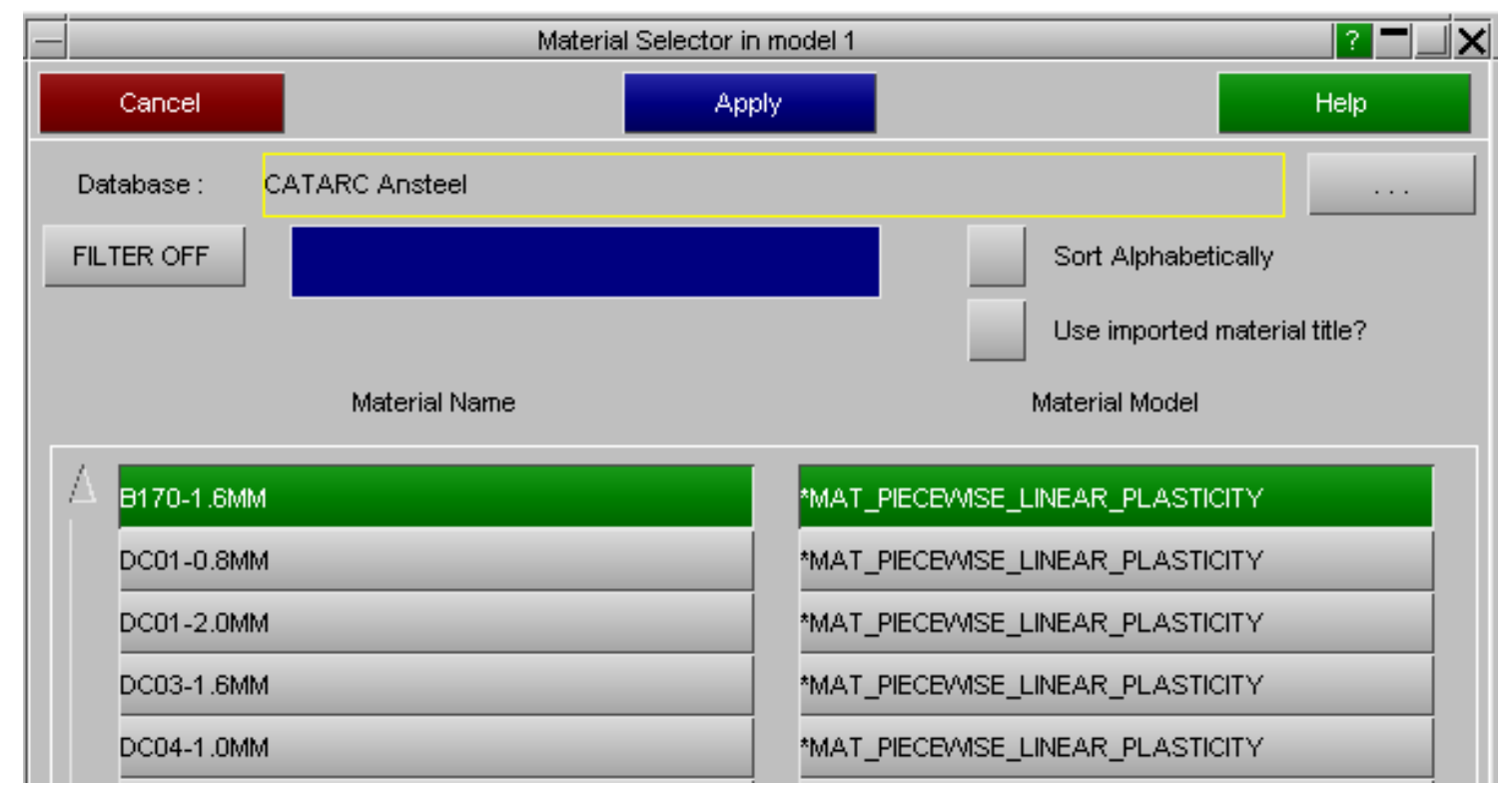

Fig. 8 CATARC Anshan steel material database. 


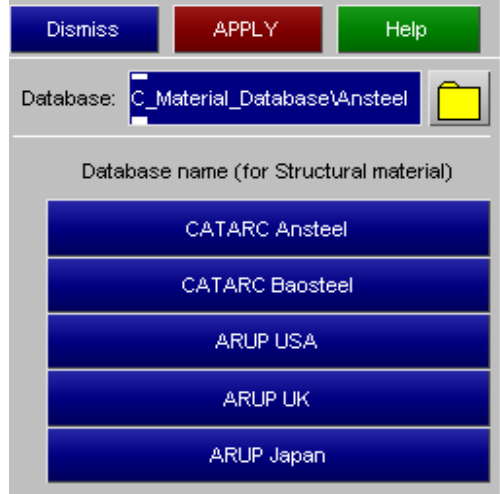

Fig. 9 Different material database switch.

\begin{tabular}{|c|c|c|c|c|c|c|}
\hline A & $\mathrm{B}$ & & c & D & $E$ & $\mathrm{~F}$ \\
\hline $\mathrm{PID}$ & CAD part no. & 7 & Part description & Section ID & Material title & Gauge \\
\hline 20300 & & $\mathrm{OB}$ & - hood & 20300 & Dco4 & 0.7 \\
\hline 20301 & & $\mathrm{OB}$ & - hood hinge 1 & 20301 & SPCC & 3.5 \\
\hline 20302 & & $\mathrm{OB}$ & - hood hinge 2 & 20302 & SPCC & 3 \\
\hline 20303 & & $\mathrm{OB}$ & - hood - I & 20303 & $\operatorname{pcol}$ & 0.8 \\
\hline 20304 & & OE & - hood reinfor & 20304 & pcol & 0.75 \\
\hline 20305 & & $\mathrm{OB}$ & - hood reinfor & 20305 & OBAL & 2 \\
\hline 20306 & & $\mathrm{OB}$ & - hood reinfor & 20306 & O8AL & 1.7 \\
\hline 20307 & & $\mathrm{OB}$ & - hood lip & 20307 & pco4 & 1.48 \\
\hline 20308 & & $\mathrm{OB}$ & - hood reinfor & 20308 & OSAL & 2 \\
\hline 20309 & & $\mathrm{OB}$ & - hood hinge 1 & 20309 & SPCC & 3.5 \\
\hline
\end{tabular}

Fig. 10 Model BOM pour and its information.

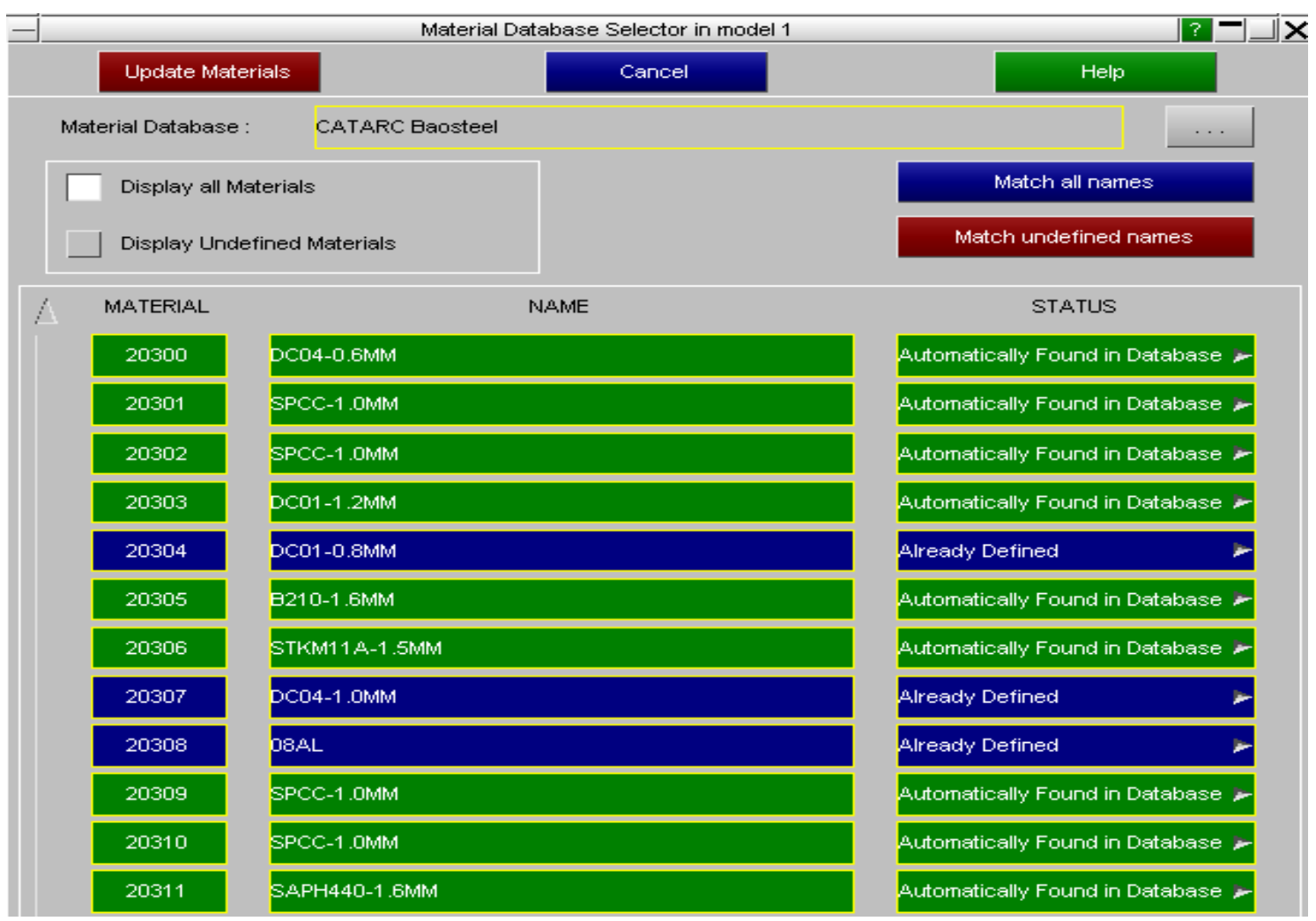

Fig. 11 Automatic matching material database. 
And then select import in material, select the appropriate database, then use the Match all names option, primer can match all of the material models automatically and the found material displayed on green background, as shown in Fig. 11, for example, the results of automatically matching by using CATARC Baosteel database.

The unmatched material may be due to not contained or naming problems or has been defined, if necessary, you can manually select alternative materials directly, so that you can built all of the material models of a model soon.

\section{Conclusions}

CATARC develops a standard material database by using more accurate post-processing methods, and the major domestic steel products, the OEM commonly used steel are included. It can help to improve the accuracy of CAE analysis effectively. At the same time, using Oasys/Primer management tools that can keep the material data consistency, thus all engineers use the same library file every time, errors will not occur in material database. And in this way, the needs of batch processing and the requirement of automation have been met, resulting in saving a lot of time to product design.

\section{References}

[1] Meng, X. M., Xie, S. G., Fang, R., and Sheng, H. 2015. "Dynamic Deformation Behavior of B340-590DP Steel Sheet.” Journal of Iron and Steel Research 27 (6): 51-5.

[2] Zhang, S., Meng, Q. Z., Xie, S. G., Fang, R., Meng, X. C., and Meng, X. M. 2016. "Dynamic Mechanical Behavior and Constitutive Model of B250P1 Low Alloy Steel.” Journal of Physical Testing and Chemical Analysis Part A: Physical Testing 52 (6).

[3] Liu, J., Yang, L. M., Xie, S. G., Hao, W. U., Zhang, S., Huang, Y. F., et al. 2017. "Dynamic Tensile Properties and Constitutive Modeling of 6061 Aluminum Alloy.” Material for Mechanical Engineering 41 (3): 49-53.

[4] “LS-DYNA® Keyword User’s Manual, Volume I R8.0.” Livermore Software Technology Corporation (LSTC): Livermore, CA 94551-5110, USA, 2015.

[5] "LS-DYNA® Keyword User's Manual, Volume II Material Models R8.0.” Livermore Software Technology Corporation (LSTC): Livermore, CA 94551-5110, USA, 2015.

[6] “Oasys ${ }^{\circledR}$ PRIMER12.1 User Manual.” Ove Arup \& Partners Ltd: Solihull, UK, 2015. 